\title{
THE SPECTRAL CLASSIFICATION OF SOUTHERN CARBON STARS
}

\author{
P. M. RYBSKI \\ Lindheimer Astronomical Research Center, Northwestern University, \\ Evanston, Ill., U.S.A.
}

\begin{abstract}
Well-widened objective prism spectra of carbon stars south of $-25^{\circ}$ declination have been examined to determine their suitability for classification. These spectra, taken by Henize in the early 1950's from South Africa as part of the Michigan-Mt. Wilson H $\alpha$ survey of the southern sky, have a reciprocal dispersion at $\mathrm{Na}$ I $5890 \AA$ of $300 \AA \mathrm{mm}^{-1}$ and are in good focus in the range between 5100 and $6600 \AA$.

Compared with spectra of northern carbon stars taken by the writer at the Lindheimer Astronomical Research Center with the one meter reflecting telescope and Cassegrain spectrograph at a grating reciprocal dispersion of $263 \AA \mathrm{mm}^{-1}$, and compared with spectra taken by Sanford of carbon stars overlapping the Henize sample, the objective prism spectra have been found of sufficient quality to support their classification into five groups following the scheme as proposed by Keenan and Morgan in 1941 and as refined by Gordon in 1967.

The first group exhibit weak $\mathrm{C}_{2}$ and $\mathrm{CN}$ features and $\mathrm{H} \alpha$ in absorption; the second, features attributable to a low $\mathrm{C}^{12} / \mathrm{C}^{13}$ ratio; the third, strong $\mathrm{C}_{2}$ and $\mathrm{CN}$ features and only moderately strong $\mathrm{Na} \mathrm{I}$ in absorption; the fourth, very strong $\mathrm{Na} \mathrm{I}$ in absorption; and the fifth, $\mathrm{H} \alpha$ in emission. Examples are given of each group, stars not fitting well into any of these groups are discussed, and the significance of each group is mentioned in light of work by Bouigue, Gordon, Peery, and Richer.
\end{abstract}

\section{Observational Material}

Between September, 1949 and August, 1951, Henize, working at the LamontHussey Observatory in Bloemfontein, South Africa, completed the Michigan-Mt. Wilson $\mathrm{H} \alpha$ survey of the sky south of declination $-30^{\circ}$. Plates were taken also of the Milky Way up to declination $-8^{\circ}$, and several plates were taken of a region in Cygnus extending as high as $+42^{\circ}$ and as low as $+15^{\circ}$. The instrument employed in the survey was the Mt. Wilson 10-in. camera corrected for the red and used with a 15-deg prism. This system yielded a plate scale of $159^{\prime \prime}$ per $\mathrm{mm}$ and a reciprocal dispersion at plate center of $300 \AA \mathrm{mm}^{-1}$ at Na I $5890 \AA$. Under conditions of good seeing, the resolution element at $6000 \AA$ near plate center approached $10 \AA$. Plate size $=14^{\prime \prime} \times 14^{\prime \prime}$ $\approx 7^{\circ} \times 7^{\circ}$ usable.

Several different filters were employed in photographing any one region to reduce the overlap between spectra while providing the maximum amount of information about any one object. Plate overlap was chosen so that any one object would fall at least once within the central one-half of a plate, and all plates were taken within $2.5 \mathrm{~h}$ of the meridian to minimize the effect of atmospheric refraction on the widening of the spectra. Most plates were calibrated with a spot sensitometer to enable later spectrophotometric reduction. The emulsion employed in the survey was Kodak 103a-E. and all plates were developed in Kodak D-19 for 4 min with continuous agitation.

Though intended for the discovery of objects exhibiting $\mathrm{H} \alpha$ in emission, the survey 
also provided spectra of objects exhibiting spectral peculiarities between 5000 and $6600 \AA$. Because the moderate dispersion in the red resulted in the spectra near plate center being $2.5 \mathrm{~mm}$ in length from 5635-6600 $\AA$, and owing to the careful and extensive widening of the spectra - being $0.5 \mathrm{~mm}$ on the average -, the spectra of $\mathrm{M}, \mathrm{S}$ and $\mathrm{C}$ stars appeared of sufficient quality to support some form of classification. The accuracy any such classifications could approach would be limited by the small number of exposures of different lengths that were made of any one field - specifically, 8,120 , and $240 \mathrm{~min}$ - and by the fact that plates of less than excellent quality were not retaken. Simon (1964) has investigated the suitability of the M star spectra for a rough temperature and luminosity classification, and Henize (unpublished) has classified the S stars on a scheme proposed by Keenan (1954). The purpose of this paper is to present the results of the writer's attempts since the spring of 1970 to place the carbon stars into some scheme of classification.

\section{History of Classification Schemes}

The historical background for the scheme proposed in this paper begins with Shane's (1928) reworking of the Harvard R-N scheme. Shane's system was based upon estimates in slit spectra, taken in the blue, of $\mathrm{C}_{2}$ and $\mathrm{CN}$ molecular band strengths. Despite the difference between the Harvard criteria and Shane's, he found it convenient to retain the R-N distinction wherein, for an equal $\mathrm{C}_{2}(1,0) 4737 \AA$ absorption intensity, the $\mathrm{N}$ star would always be much fainter in the ultraviolet than the $\mathrm{R}$ star. When Shane examined the $\mathrm{N}$ sequence separately and attempted to relate the differences in spectral intensity maxima to differences in blackbody temperatures, often he derived temperatures which seemed too low to believe. Then current and later radiometric measures of four such stars by Pettit and Nicholson $(1928,1933)$ suggested the range in carbon star temperatures to be from $2000-2400 \mathrm{~K}$. The problem of how to relate the $\mathrm{R}$ to the $\mathrm{N}$ sequence was complicated by Shane's finding that in each sequence the $\mathrm{C}_{2}(1,0)$ band passed through a maximum while the ultraviolet cyanogen bands appeared to pass through a maximum only in the $\mathrm{R}$ sequence. The similarity between the sequences was underscored by Wurm's (1932) showing that by placing the stars in an order determined by a color index defined between the ultraviolet and infrared portions of the spectrum the sequences were intermingled at similar index values.

The first significant improvement in carbon star classification after Shane was that proposed by Keenan and Morgan in 1941. Based primarily on atomic line ratios in blue spectra of reciprocal dispersion $125 \AA \mathrm{mm}^{-1}$ at $\mathrm{H} \gamma$ - line ratios found sensitive to temperature in $K$ and $M$ stars - and secondarily on the $C_{2}(0,1) / C_{2}(1,2)$ bandhead ratio, the absolute strength of the unresolved $\mathrm{Na}$ I doublet at $5890 \AA$, and continuum measures in the green, orange and red portions of the spectrum, this scheme proposed merging the $\mathrm{R}$ and $\mathrm{N}$ Harvard branches into a single sequence with the early $\mathrm{R}$ 's placed largely from $\mathrm{C} 0-\mathrm{C} 3$ and with the later R's and all N's placed from $\mathrm{C4}-\mathrm{C}$. They also defined a second dimension - carbon abundance - determined from eye estimates of the absolute strength of the $C_{2}(0,1)$ bandhead at $5635 \AA$. Keenan and 
Morgan recognized that this strength would be affected not only by temperature but also possibly by a spread in luminosity and that it would have to be studied in greater detail than was possible for them before its abundance-dependent character would become clear.

The principal weakness of the Keenan-Morgan system as defined was that, for stars defining the system later than $\mathrm{C} 4$, blue spectra usually could not be obtained. Consequently, their arrangement by temperature had to be based primarily on the three red criteria mentioned above, and each criterion had its own difficulties. The continuum measures were chosen in places in the red spectra, of reciprocal dispersion $250 \AA \mathrm{mm}^{-1}$ at $6100 \AA$, where it appeared there were few disturbing molecular features. Subsequent research at higher dispersions has shown that only one of these regions - $5670 \AA-$ is undisturbed. Secondly, the absolute strength of the $\mathrm{Na}$ I line had to be corrected for the overlying $\mathrm{CN}(7,2)$ absorption, a procedure even Keenan and Morgan considered qualitative at best (Keenan, 1971b). Finally, while the use of the ratio of the first two members of the $C_{2}(0,1)$ band sequence was based on the laboratory work of Wurm (1932) - showing the ratio changing from near unity at $3000 \mathrm{~K}$ to 3 at $1500 \mathrm{~K}$-, the whole band sequence was contaminated by the $\mathrm{CN}(5,0)$ band. Since the structure of this band, its intensity and complexity is every bit as considerable as the $\operatorname{CN}(7,2)$ band, ${ }^{*}$ any variation in $\mathrm{CN}$ strength - such as the writer has encountered in at least two $\mathrm{CH}$ stars - could introduce systematic errors into a temperature sequence based upon the $C_{2}(0,1) /(1,2)$ ratio. What resulted, then, was a scheme which at best was heterogeneous in principle and which at worst showed large scatter for stars classified later the C4 (Morgan, 1971).

Three major rediscussions of carbon star classification have appeared since 1941 . Bouigue (1954) proposed a scheme based largely on spectra of a dispersion similar to that used by Keenan and Morgan, making use of the absolute strength of $\mathrm{Na} \mathrm{I}$ corrected for the overlying $\mathrm{CN}(7,2)$ band while utilizing ratios of the unresolved band structure of the +5 and $+4 \mathrm{CN}$ band sequences and the -1 and -2 band sequences of $\mathrm{C}_{2}$ as further measures of temperature. Bouigue's results corroborate roughly those of Keenan and Morgan, while at the same time isolating for special consideration a subclass $\mathrm{J}$ of carbon stars showing an additional, apparently molecular absorption feature at $6270 \AA$ which Sanford (Keenan and Morgan, 1941) found correlated inversely in strength with increasing apparent $\mathrm{C}^{12} / \mathrm{C}^{13}$ ratio.

Gordon (1967) re-examined the classification problem from the perspective not only of three times greater dispersion in the red than employed by Keenan and Morgan but also of blue spectra of the brighter stars of all carbon types. By examining blue spectra of $K$ and $M$ giants in the region $4300-4500 \AA$, she defined additional atomic line ratio criteria for temperature. When these were applied to the carbon star spectra, three conclusions could be drawn about the Keenan-Morgan scheme. First, the order of the stars from $\mathrm{C} 0-\mathrm{C} 3$ was preserved, a result for which one would have hoped, this interval being that for which Keenan and Morgan had adequate blue spectral data.

* Marenin, 1971. 
Secondly, those stars classified at $\mathrm{C} 4$ - and at least one at $\mathrm{C} 5$ - which exhibited features suggestive of a much lower $\mathrm{C}^{12} / \mathrm{C}^{13}$ ratio than the average for carbon stars, and all of which belonged to Bouigue's subclass $\mathrm{J}$, should have temperatures equivalent to middle $\mathrm{K}$ through early $\mathrm{M}$.

Thirdly, the stars between types C5 and C9 divided into three groups with the following characteristics. First, there were those showing only weak to moderately strong $\mathrm{Na}$ I with $\mathrm{CN}$ and $\mathrm{C}_{2}$ absorption strengths varying inversely with the $\mathrm{Na}$ I strength. Secondly, there were a group of stars exhibiting extremely strong $\mathrm{Na}$ I while showing only moderately strong $\mathrm{CN}$ and usually weak $\mathrm{C}_{2}$ features and which had been found by other observers to exhibit features peculiar even for carbon stars. Thirdly, there were the carbon stars exhibiting $\mathrm{H} \alpha$ in emission. From her blue spectra and from the photometry of Mendoza and Johnson (1965), Gordon found that in these cool carbon stars the $\mathrm{Na}$ I absorption strength was not always a trustworthy criterion of temperature. Those stars with extremely strong $\mathrm{Na}$ I were found to merge into the group in which $\mathrm{Na}$ I was only weak to moderately strong. And those stars with $\mathrm{H} \alpha$ in emission appeared to be the coolest of all, despite $\mathrm{Na}$ I strengths which would have merged them randomly into the group with weak to moderately strong $\mathrm{Na}$ I strengths. All in all, the temperature range in these cool carbon stars appeared to extend from M3.5-M7.

Comprised of all $\mathrm{N}$ stars and one R9 star, the three groups of cool carbon stars seemed to have one feature in common: a significantly enhanced Ba II line at $4554 \AA$. Gordon (1968) took this as evidence for these stars having enhanced abundances of $s$-process elements and as one of several reasons for associating them generically with the $\mathrm{Ba}$ II stars (Bidelman and Keenan, 1951), which are of higher temperature than the cool carbon stars, rather than with the $\mathrm{R}$ stars of type 8 and earlier. Not considered carbon stars despite their exhibiting a stronger $C_{2}(0,0)$ absorption than is considered normal in the oxygen sequence stars (Bidelman, 1956), the Ba II stars are known to be rich in s-process elements (Burbidge and Burbidge, 1957; Warner, 1965). Her suggestion of associating the $\mathrm{Ba}$ II with the $\mathrm{N}$ sequence has received support from abundance analyses by Utsumi (1967) of five typical N stars, each of which were shown to possess enhanced $s$-process abundances. Gordon (1967) also found one R9 star - HD 59643 to have an enhanced $\mathrm{Ba}$ II line. More work needs to be done on the very late $\mathrm{R}$ stars before it will be clear whether they belong to the $R$ or to the $B a$ II-N sequence.

Gordon (1968) implies, without stating so specifically, that all N stars should be considered to have enhanced $s$-process abundances. Her evidence for such a conclusion is that every one of her $14 \mathrm{~N}$ stars for which she had a blue spectrum showed the enhanced $\mathrm{Ba}$ II line. And since these stars have a unique red spectral appearance which sets them aside from all but the latest $\mathrm{R}$ stars and from the subclass $\mathrm{J}$ stars, one might be tempted to call all $\mathrm{N}$ stars 'late Ba II stars' solely on the basis of this unique red appearance. However, her implication may not be proved conclusively by 14 spectra. Assignment of cool carbon stars to the late Ba II class may require a blue spectrum for each one so assigned - an observational problem of considerable difficulty. Until such a study is 
completed, the $\mathrm{N}$ and late $\mathrm{R}$ stars should probably be referred to merely as the 'cool carbon stars', a convention adopted in this paper.

A blue spectral study of these cool carbon stars may well affect the study of the optical spiral structure of our Galaxy. When Peery (1970) sorted the N stars by their light variability; assigned them absolute visual magnitudes by forcing them into the spiral arms defined by young clusters, $H$ II regions and Cepheids more luminous than $M_{\mathrm{v}}=-4.3$; and corrected their mean magnitudes for interstellar absorption; then he found the mean absolute visual magnitude of 26 irregularly varying $\mathrm{N}$ stars to be -3.5 with only a small scatter about this mean. Such a small scatter in the mean is acceptable, says Peery, because spectrally and photometrically these stars are so similar. In short, Peery feels the irregular $\mathrm{N}$ variables may be used to trace optical spiral structure. One of the results of the classification of the Henize carbon stars will be having brought research one step closer to a large-scale Peery-like treatment of southern $\mathrm{N}$ irregular variables, since, first, the cool carbon stars stand out so clearly from the hotter ones, and, secondly, because among the cooler stars those potentially of Mira-type can be sorted out from those which are semiregular and irregular in their light variability.

Two additional reports deserve mention since they relate directly to the scheme of objective prism classification presented below. The first is the work by Yamashita (1967) in which he gathers together plate material on carbon stars accumulated over $20 \mathrm{yr}$ at Victoria by McKellar - blue and red spectra of generally higher dispersion than that used by Keenan and Morgan. First, from his own study of $\mathrm{K}$ and $\mathrm{M}$ stars, Yamashita defines blue criteria which he then applies to carbon stars of all varieties, confirming generally Keenan and Morgan's results for all stars save those exhibiting $\mathrm{Na} \mathrm{I}$ in great strength. Secondly, he extends what is essentially the Keenan-Morgan system to many carbon stars without a previous Keenan-Morgan type.

The second report is that by Richer (1971) on a scheme of temperature classification employing the infrared $\mathrm{CN}$ bandheads at 7852, 7876 and $7899 \AA$ and the infrared $\mathrm{Ca}$ II triplet at 8498,8543 and $8662 \AA$ in spectra of reciprocal dispersion $124 \AA \mathrm{mm}^{-1}$. A comparison (see Figure 8) of Richer's types with Mrs Gordon's unpublished blue types shows a surprising lack of correlation for the cool carbon stars for which Richer's scheme could have provided a valuable means of temperature discrimination. Gordon's hotter carbon stars do fall at earlier Richer type, while the cooler ones cluster largely at Richer type C5II. It remains, then, an unsolved problem why cool carbon stars, which vary between Gordon's equivalent blue type M3.5 to M7, show little or no temperature dispersion in the Richer system. (The error bars in Figure 8 were provided by Gordon (1971) and represent $75 \%$ confidence limits. Richer makes no star-by-star estimate of classification error, stating his error on the average as plus or minus one subclass.)

Richer's most significant contribution seems his having found an apparent dispersion in luminosity for the carbon stars. This dispersion makes its appearance through a general washing-out of the infrared molecular features and an enhancement of infrared atomic lines isolated by Keenan and Hynek (1945) as sensitive to luminosity in $\mathrm{M}$ 
stars. In the group of stars to which Richer assigns a higher luminosity are found a surprising number of stars which Bouigue either did or would have assigned to his subclass $\mathbf{J}$. If one believes the higher temperatures Gordon assigns to these stars, then the enhancement of the luminosity-sensitive lines in them may only be an effect of their higher temperature. Clearly, further research seems justified to sort out in Richer's class I which effects may be attributed to temperature and which to luminosity. Identifying additional stars of subclass $\mathbf{J}$ would aid in the solution of this problem. It is one of the features in the classification scheme proposed below that carbon stars of subclass $\mathrm{J}$ form a separate class.

\section{The Objective-Prism Classification Scheme}

Difficulties in classifying the Henize spectra were encountered immediately. Since these spectra resembled most closely in dispersion the spectra reproduced in Keenan and Morgan's (1941) article, a comparison between the two was made only to reveal that the Henize spectra contained far more information longward of $6000 \AA$ than did the reproductions of Keenan and Morgan's. When Mrs Gordon's reproductions (1967) were compared to the Henize sample, her spectra were found of too high a dispersion for direct comparison. Finally, of the few Henize spectra north of declination $-15^{\circ}$, most were too faint to have had any previous classification. This made impossible the direct comparison of Henize spectra which might have had some form of classification to Henize spectra without any types.

An attempt was made at using slit spectra obtained by Sanford for some kind of comparison and analysis, but little success was encountered owing to their widely varying resolution, dispersion and degree of exposure. Only Sanford's spectrum of HD 75021 proved useful, for it contained broad features which could also be seen in the Henize spectrum of the same star, features which have since been used to select out subclass $\mathrm{J}$ stars from the Henize sample.

Even though individually the Sanford spectra were not of aid in solving the classification problem, they pointed the way toward the use of broad features in spectra similar but not identical to the Henize spectra in their instrumental quality and which could be taken of as many stars in the north as would be necessary to sort out the Henize sample. To this end, the writer has secured some 185 spectra of 103 northern carbon stars brighter than $m_{v}=10.0$ at the Lindheimer Astronomical Research Center with the one meter reflector and Cassegrain spectrograph. The reciprocal dispersion of these spectra is $263 \AA \mathrm{mm}^{-1}$, the projected slit width is $6 \AA$, and all the spectra were widened to $0.62 \mathrm{~mm}$. The resulting length-to-width ratio of these grating spectra is nearly identical to that found in most of the Henize spectra. All spectra were exposed to similar densities at $5670 \AA$ to facilitate intercomparing $\mathrm{Na} \mathrm{I}$ intensities and were taken on Kodak IIa-F plates to minimize having to take more than one spectrum of a given star to insure proper exposure between 5635 and $6600 \AA$. All plates were developed in Kodak D-76 at $20^{\circ}$ Centigrade for 15 min with continuous, vigorous agitation in a large tray rocked by an International Observatory Instruments tray 
agitator to insure not only properly but also reproducibly developed spectra.

From this sample, coarse criteria were devised which placed these and the Henize spectra into seven groups, the first five of which were suggested by the major groups in Mrs Gordon's scheme, while the sixth contained peculiar stars with carbon characteristics and the seventh held those stars which might possibly appear like carbon stars at some time during their variability. Examples of each of these groups is given in Figures 1 through 7. With the exception of the stars HD 91708, Co.D. $-62^{\circ} 466$, and those stars with a catalog name followed by a small letter $p$ - standing for prismatic dispersion - only the spectra identified by a simple sequence number were taken by Henize. Those taken by the writer are identified with a catalog name or number which may or may not be followed by a small letter $g$ - standing for grating dispersion. The use of sequence numbers to identify spectra of Henize carbon stars was necessitated by the absence for these stars of reliable position measurements which would have made possible identification and a catalog reference. These stars will be identified in a later article published jointly by Henize and the writer.

In Figures 2 and 4 examples are given of stars for which the writer has in his possession a spectrum taken by Henize and one taken by himself. These have been shown to illustrate how much detail really is retained in the Henize spectra. Where such a direct comparison was not possible, a northern grating spectrum was chosen to match a southern prismatic spectrum to illustrate features in the southern spectrum which can also be seen - and whose source can be identified - in the northern.

\subsection{The EARly R StARS. KeENAN AND MORGAN TyPeS C0-C3. FIGURE 1}

These stars always exhibit $\mathrm{H} \alpha$ in absorption if $\mathrm{H} \alpha$ is present at all. $\mathrm{Na}$ I shows weakly in absorption as does the $\mathrm{C}_{2}(0,1)$ band sequence. If the $\mathrm{C}_{2}(0,2)$ band sequence is present, it is usually very weak. The $\mathrm{CN}(4,0)$ band sequence may be present, but it is considerably weaker here than in any other class save the subclass $\mathbf{J}$ stars.

The stars chosen to illustrate this group are the northern R3 star HD 5223, for which a grating spectrum is shown, and the southern R0 star HD 91708 shown in a prismatic spectrum. Both are rather featureless except for the $C_{2}(0,1)$ band sequence and a weak $\mathrm{Na} I$ line. Owing to this featurelessness of the early $\mathrm{R}$ stars, these were the most difficult to detect and classify on the Henize plates. It is doubtful any stars not already in the Henry Draper survey as early R stars, will be found in the Henize sample.

Please note that marked on the spectrum of HD 5223 are the yellow and green lines of mercury vapour from the street lamps of Chicago. These lines are marked in each grating spectrum in other figures where they are sufficiently intense to be mistaken for part of the stellar spectrum.

\subsection{THE SUBCLASS ' $J$ ' STARS. KEENAN AND MORGAN TYPE C4. FIGURE 2}

Most stars in this group exhibit not even a trace of the $\mathrm{CN}(4,0)$ band sequence. Instead, the region between 6191 and $6600 \AA$ is marked by the absorption band at $6270 \AA$ used by Bouigue to define this class and by a triplet of dark bands - a pair between the expected positions of $\mathrm{CN}(5,1)$ and $(6,2)$ and a single dark band just 


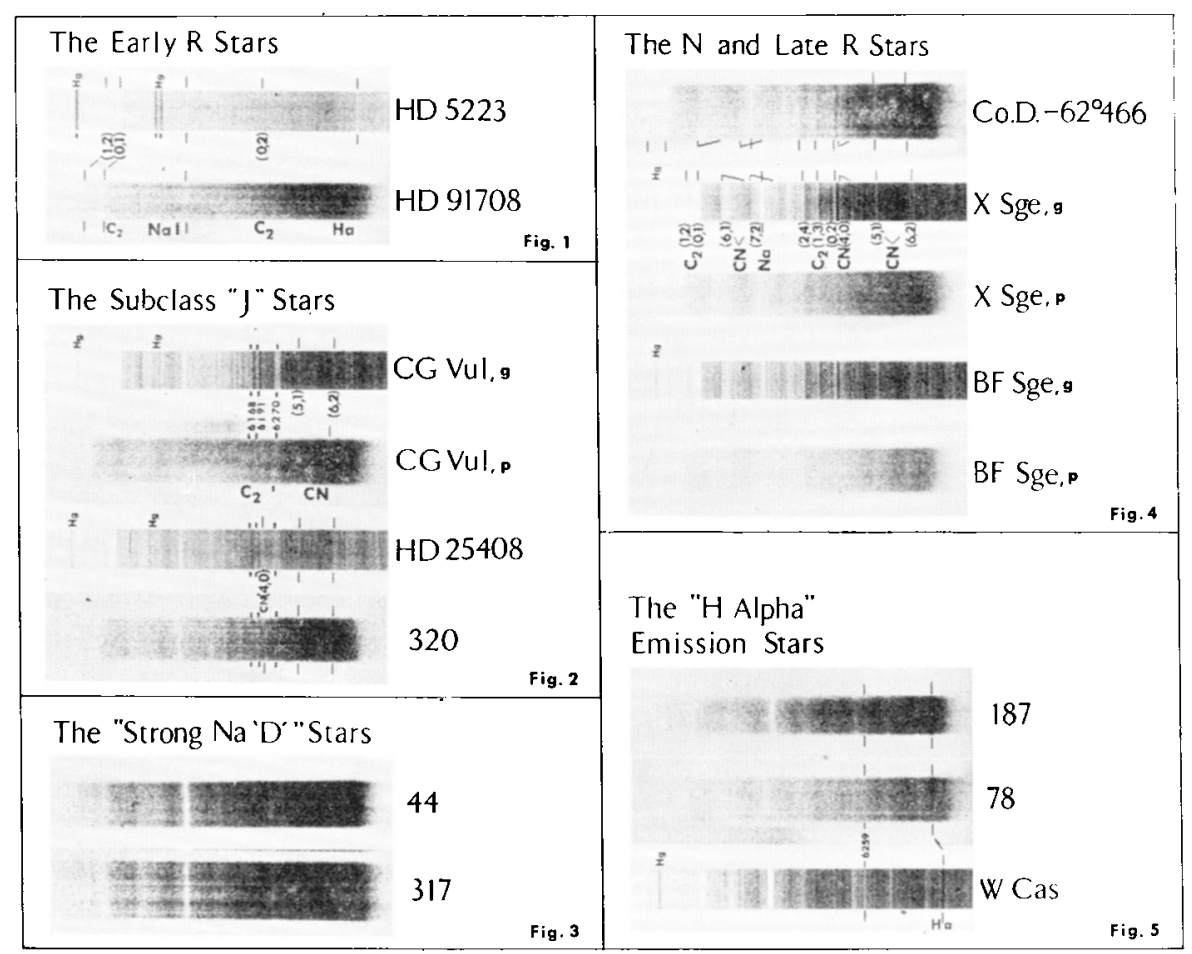

Figs. 1-5.

blueward of the expected position of $\mathrm{H} \alpha$. The regions immediately redward of $\mathrm{C}^{12} \mathrm{C}^{12}$ $(0,2) 6191 \AA$ and $C^{12} C^{13}(0,2) 6168 \AA$ are each marked by dark narrow bands of equal strength. These bands will be seen in stars of later type, but the band at $6168 \AA$ never approaches the intensity of the one at $6191 \AA$ as it does in this class. Similar dark bands each redward of the $C^{12} C^{12}$ and $C^{12} C^{13}(1,3)$ and $(2,4)$ bandheads may appear and only in this class. $\mathrm{Na} \mathrm{I}$ may be absent, but it may also be weak to moderately strong. But it never approaches the intensity reached in any of the normal cooler stars. When the $\mathrm{CN}(5,0)$ band sequence can be seen, $(6,1)$ appears most strongly while $(7,2)$ and $(8,3)$ seem washed out. Finally, on the E plates, the continuum redward of $5635 \AA$ appears steplike. The portion blueward of $6191 \AA$ is always uniformly fainter than the portion redward of that point.

Two extremes of this class are illustrated in Figure 2. The northern subclass $J$ star CG Vul shows very strongly the features characterizing the group. Note the strength of the $6270 \AA$ band, the strengths of the dark bands in the $C_{2}(0,2)$ band sequence, and the overall weakness of the $\mathrm{CN}(4,0)$ band sequence. This should be compared to the northern star HD 25408 and to the southern star Henize 320 . In both, the $\mathrm{CN}(4,0)$ band sequence is much stronger than in CG Vul, and the $6270 \AA$ is much reduced in strength. In fact, Henize 320 represents a limit detection problem in this class. 


\subsection{The STRONG Na 'D' STARS. KEENAN AND MORGAN TYPES C8-C9. FIGURE 3}

Stars included in this group were selected almost entirely on the basis of their extraordinarily strong $\mathrm{Na}$ I absorption. Some exhibited additional peculiarities, such as the dark bands at 6191 and $6168 \AA$ typical only of a subclass $J$ star. More often, they exhibited very weak $C_{2}$ features but readily visible $C N$ features. It is not clear these stars should form a group apart from the peculiar stars discussed later. Only a small number of Henize stars fall into this group, yet the strength of their $C_{2}(0,1)$ bandhead and the presence of an absorption at $6259 \AA$ - characteristic of stars of apparently low temperature - justifies temporarily their separate consideration.

\subsection{The N AND LATE R STARS. KEENAN AND MORGAN TYPES C5-C9. FIGURE 4}

To be a member of this class, a spectrum had to satisfy three criteria. The first was that all visible features had to be assignable to normal molecular and atomic sources, i.e., only the usual features due to $\mathrm{C}_{2}, \mathrm{CN}$ and $\mathrm{Na}$ I were allowed. Secondly, $\mathrm{H} \alpha$ could not be in emission. The third criterion required the following behavior of the usual molecular and atomic sources. Since in the grating spectra dark bands each redward of a normal bandhead in the $\mathrm{C}_{2}(0,2)$ band sequence were found to correlate directly in their strength with the absolute strength of the absorption bandhead of the $\mathrm{C}_{2}(0,1)$ band sequence at $5635 \AA$ (originally Keenan and Morgan's abundance index); and because it was found that in the 'normal' northern carbon stars the latter bandhead strength correlated inversely with decreasing temperature, as determined from Gordon's (1967) blue spectra, while correlating directly with the expected temperature-dependent ratio of the $(5,0)$ and $(4,0) \mathrm{CN}$ band sequences; then these dark bands - which could always be seen in the Henize spectra when the region of the $\mathrm{CN}(4,0)$ band sequence was properly exposed, a condition seldom met by the $\mathrm{C}_{2}(0,1)$ band sequence - had to correlate directly in strength with the $\mathrm{CN}$ band sequence ratio and inversely with the absolute strength of $\mathrm{NaI}$. That is, when the former two were weak, the latter had to be strong; when the former two were strong, the latter had to be weak. Only a few stars were found in the Henize sample in which this final condition could not be met, and these spectra are receiving further consideration.

The writer was fortunate to have two northern $\mathrm{N}$ stars - X and BF Sge - for which he had for direct comparison both Henize spectra of good quality and his own grating spectra. A spectrum of Co.D. $-62^{\circ} 466$ has been included in Figure 4 to show the difference between spectra taken by Henize at small zenith distance - typified by $-62^{\circ} 466$ - and spectra taken at large zenith distance - typified by the stars in Sagitta. Owing most likely to differences in average conditions of seeing, these differences make difficult or impossible direct comparisons of absorption intensities in spectra of stars north and south of the celestial equator in the Henize sample.

The important molecular and atomic absorption features seen in this type of star are identified both in the grating spectrum of XSge and in the prismatic spectrum of 
$-62^{\circ} 466$. It is easily seen from this example how different these stars are in appearance on the Henize plates from the subclass $J$ and early $R$ stars.

\subsection{The H $\alpha$ EMISSION STARS. KeENAN AND MORGAN TYPES C5-C9. FIGURE 5}

To be included in this group, a star had to exhibit only $\mathrm{H} \alpha$ in emission and either the $\mathrm{CN}$ or $\mathrm{C}_{2}$ band sequences in absorption. In the northern sky such a selection criterion would net not only bona fide carbon stars but also stars showing simultaneously features characteristic of carbon and S stars. Owing to Henize's survey favoring objects exhibiting $\mathrm{H} \alpha$ emission, many such carbon stars were detected. But many such stars were so poorly exposed blueward of $\mathrm{H} \alpha$ that it was impossible to say with certainty that they were normal or peculiar. It was felt more consistent to keep all the $\mathrm{H} \alpha$ emission stars together and to comment on peculiarities individually when seen than to divide up these stars into a 'normal' and a peculiar class.

The stars included in Figure 5 would probably fall into the 'normal' group at the dispersion of the survey. Henize stars number 187 and 78 show all the features marked for the $\mathrm{N}$ and late $\mathrm{R}$ stars in Figure 4, though somewhat less intensely, while showing an additional feature at $6259 \AA$ which Gordon (1967) attributes to a zero-volt transition of Sc $\mathrm{I}$ but which Keenan (1971b) would prefer to attribute to a confluence of low-excitation $\mathrm{Ti}$ I lines. Gordon found this line to strengthen at later equivalent blue spectral types, implying it was a temperature-sensitive line.

\subsection{The PeCuliar Stars. Figure 6}

All stars in this group have the common property of exhibiting weakly the CN $(4,0)$ band sequence, and most show evidence for the presence of the low-temperature $6259 \AA$ line. After these similarities, the stars appear to fall into two groups. The first are similar to CY Cyg in showing apparently no $\mathrm{CN}(5,0)$ band sequence and no evidence for the $\mathrm{C}_{2}(0,1)$ band sequence. A prismatic spectrum of Henize 201 compares favorably to a grating spectrum of CY Cyg obtained by the writer this past September. The second group appears similar to a recently obtained grating spectrum of WZ Cas in showing a weak but definitely present $\mathrm{C}_{2}(0,1)$ absorption, the low-temperature $6259 \AA$ line somewhat more strongly than in the CY Cyg group, and an additional, apparently molecular feature redward of $\mathrm{CN}(5,1)$ which degrades to the red and is most intense at $6379 \AA$. Examination of grating spectra in the writer's collection of $+38^{\circ} 955$, RS Cyg, and R CMi showed that the band appeared in the first two but not in the last.

One encounters confusing evidence when trying to ascribe this band to the $(1,1)$ $\mathbf{R}_{2}$ band of zirconium oxide. When the illustration in the article on GP Ori by Bidelman (1950) was examined, both GP Ori and FU Mon exhibited the band in greater strength than did either $+38^{\circ} 955$ and $\mathrm{WZ}$ Cas also shown in the article. But while both GP Ori and FU Mon seemed to show in great strength the $(0,0) R_{3}$ band of $\mathrm{ZrO}$ at $6473 \AA$, WZ Cas and $+38^{\circ} 955$ did not. The $6259 \AA$ line in WZ Cas (in the grating spectrum) seems peculiarly stronger than in any other star of accepted low temperature - such as Z Psc -, and this enhancement may be due to the $(1,1) R_{1}$ band 


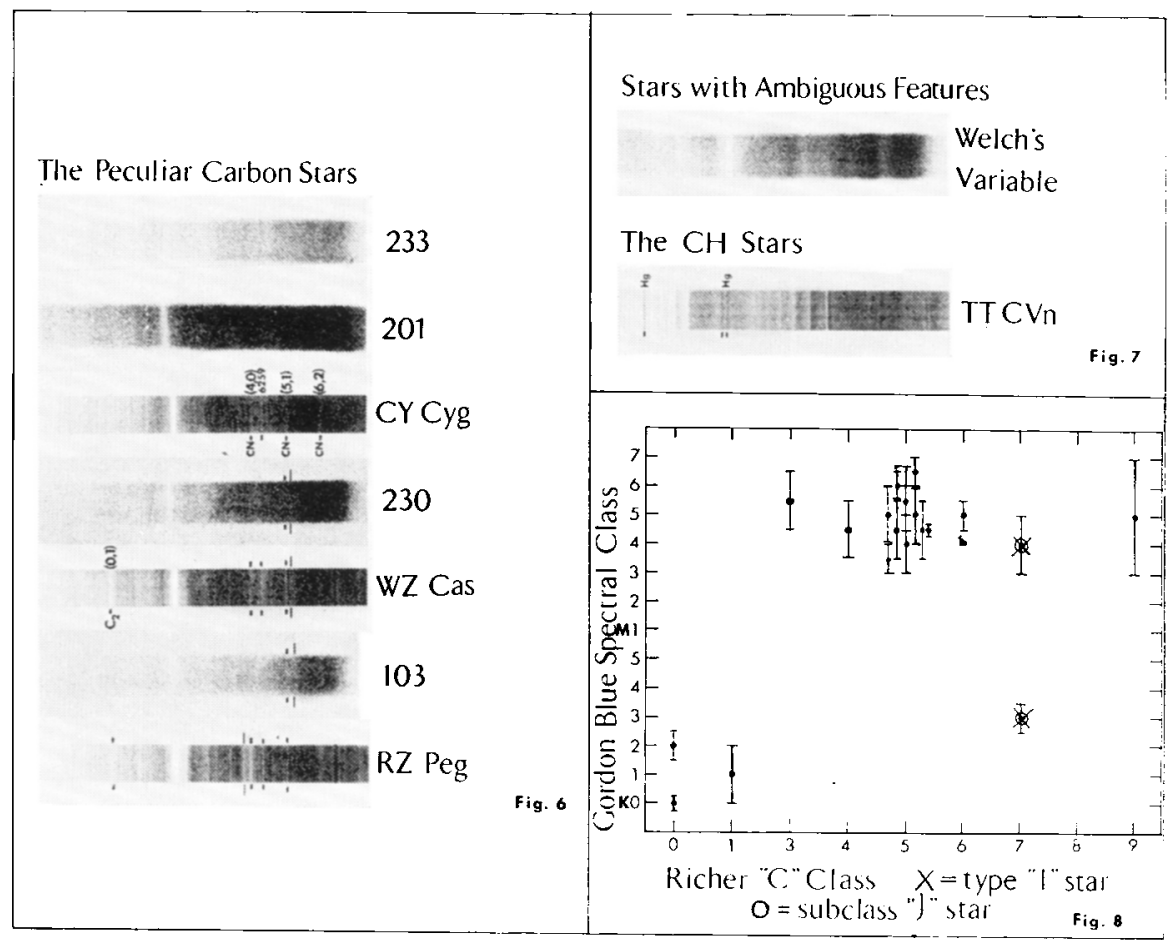

Figs. 6-8.

of $\mathrm{ZrO}$ at $6260 \AA$. But when laboratory evidence is consulted, the tentative identification of the new band with a bandhead of $\mathrm{ZrO}$ is not supported: bands which should be present along with the $(1,1) R_{2}$ band, and which should be stronger than it, are not seen in WZ Cas or $+38^{\circ} 955$. Further complicating the matter is that direct comparison of this spectrum of WZ Cas with a spectrum of the S star HR 8714 taken with the same instrument reveals the band present in the latter star but only weakly so when compared with the expected, properly stronger bands of $\mathrm{ZrO}$.

The purpose of discussing this band at length is not to argue for a particular identification but rather to point to the stars in the south which exhibit the band in the Henize spectra. Numbers 230 and 103 are shown in Figure 6 along with WZ Cas, and large tic marks are used to set off the unidentified band in all three stars as it appears just redward of $\mathrm{CN}(5,1)$ marked by smaller tic marks.

Finally, included in Figure 6 is a spectrum of RZ Peg obtained recently by the writer. Examination of the spectrum reveals not only an unexpected enhancement of what heretofore has been the $\mathrm{CN}(4,0)$ bandhead but also the presence of an additional band blueward of both $\mathrm{CN}(4,0)$ and $\mathrm{C}_{2}(0,2)$. Also, the region surrounding $\mathrm{Na}$ I looks peculiarly depressed despite the spectrum's showing hardly any evidence of the $\mathrm{CN}(5,0)$ band sequence. There is sufficient evidence from other researchers (Greene, 1971; Keenan, 1971b) that these peculiar absorptions are caused by the 
red and orange systems of $\mathrm{CaCl}$. No Henize star has been found to date with these features.

\subsection{StaRS NOT YET INCLUDED IN THE CLASSIFICATION SCHEME. FIGURE 7}

Several stars mentioned above have been referred to in the literature (Keenan, 1971a) as stars with features similar both to carbon stars and to S stars. One of these is CY Cyg which in the spectrum illustrated looks rather like a carbon star. Most of these stars are variable in spectral appearance as well as they are variable in light, and several of these stars have been known to look more like $S$ stars than carbon stars at their cooler phases. Such a spectrum variable is Welch's in Crux (Keenan, 1971a). The writer was privileged to see a spectrum of this star taken recently at moderate dispersion by Dr Keenan. At the time the spectrum was obtained, the star was about 8.5 visual magnitude; and the usual features of $\mathrm{C}_{2}$ and $\mathrm{CN}$ could be made out, though they were probably weaker than in the normal $\mathrm{N}$ stars. Included in Figure 7 is a Henize spectrum of this variable when the star was about 10 visual magnitude, and it is easily seen to bear little resemblance to any other spectrum shown in this article. This star was not included by Henize in his list of potential carbon stars because it appeared more like an $S$ star than like a carbon star. Had the $C_{2}(0,1)$ bandhead been visible instead of being obscured by the spectrum of another star, this star might have found its way into the list of carbon stars. Suffice it to say that the writer has not included stars such as Welch's variable in the Henize list unless $C_{2}(0,1)$ is clearly present.

Finally, spectral peculiarities can often be confused with poor resolution in the Henize spectra. Such would probably be the conclusion if a spectrum like that of the northern $\mathrm{CH}$ star TT CVn were seen in the sample. This is because both the $\mathrm{CN}(4,0)$ and $(5,0)$ band sequences are so weak as to reveal an almost pure $C_{2}$ absorption spectrum, making the star look like an early $\mathrm{R}$ star. It is not clear at the moment whether such stars will find their way into a separate class or accidentally be misclassified.

\section{Acknowledgements}

The writer would like to express his extreme gratitude to Dr Karl Henize for placing at the writer's disposal his valuable plate collection and for invaluable assistance in approaching the classification problem: Special thanks must be extended to $\mathrm{Dr}$ W. Buscombe under whom the writer is using the material discussed for his thesis and to Dr J. Allen Hynek for scheduling the excellent observing facilities of the Lindheimer Astronomical Research Center for obtaining the northern slit spectra. Finally, the writer owes a special debt of gratitude to Drs P. C. Keenan, W. W. Morgan, and C. P. Gordon for their encouragement and private communications concerning their classification schemes. This work was completed while the writer held an NDEA Title IV fellowship.

\section{References}

Bidelman, W. P.: 1950, Astrophys. J. 112, 219.

Bidelman, W. P.: 1956, Vistas in Astronomy 2, 1428. 
Bidelman, W. P. and Keenan, P. C.: 1951, Astrophys. J. 114, 473.

Bouigue, R.: 1954, Ann. Astrophys. 17, 104.

Burbidge, E. M. and Burbidge, G. R.: 1957, Astrophys. J. 126, 357.

Gordon, C. P.: 1967, Ph. D. Thesis, University of Michigan.

Gordon, C. P.: 1968, Astrophys. J. 153, 915.

Gordon, C. P.: 1971, private communication

Greene, A. E.: 1971, Ph. D. Thesis, Ohio State University.

Keenan, P. C.: 1954, Astrophys. J. 112, 219.

Keenan, P. C.: 1971a, Monthly Notices Roy. Astron. Soc. 153, short communication.

Keenan, P. C.: 1971b, private communication.

Keenan, P. C. and Hynek, J. A.: 1945, Astrophys. J. 101, 265.

Keenan, P. C. and Morgan, W. W.: 1941, Astrophys. J. 94, 501.

Marenin, I.: 1971, private communication.

Mendoza, E. E. V. and Johnson, H. L.: 1965, Astrophys. J. 141, 161.

Morgan, W. W.: 1971, private communication.

Peery, B.: 1970, Kitt Peak Symposium of Late-type Stars.

Pettit, E. and Nicholson, S. B.: 1928, Astrophys. J. 68, 279.

Pettit, E. and Nicholson, S. B.: 1933, Astrophys. J. 78, 320.

Richer, H. B.: 1971, Astrophys. J. 167, 521.

Shane, C. D.: 1928, Lick Obs. Bull., 13, 123.

Simon, L. W.: 1964, M.S. Thesis, Northwestern University.

Utsumi, K.: 1967, Publ. Astron. Soc. Japan 19, 342.

Warner, B.: 1965, Monthly Notices Roy. Astron. Soc. 129, 263.

Wurm, K.: 1932, Z. Astrophys. 5, 260.

Yamashita, Y.: 1967, Publ. Dominion Astrophys. Observ. 13, no. 5, 67. 